\title{
Modeling of Plane Arrays Using a Variational Approach
}

\author{
Mykhaylo I. Andriychuk' ${ }^{1}$ Yarema F. Kuleshnyk ${ }^{2}$ \\ ${ }^{1}$ Department of Numerical Methods of Mathematical Physics, Pidstryhach Institute for Applied Problems of Mechanics \\ and Mathematics, NASU, Lviv, Ukraine \\ ${ }^{2}$ Department of Informatics, Lviv State University of Internal Affairs, Lviv, Ukraine \\ Email: andr@iapmm.lviv.ua, kuleschnyk@gmail.com
}

How to cite this paper: Andriychuk, M.I. and Kuleshnyk, Ya.F. (2018) Modeling of Plane Arrays Using a Variational Approach. Open Journal of Antennas and Propagation, 6, 93-105. https://doi.org/10.4236/ojapr.2018.64009

Received: November 28, 2018 Accepted: December 26, 2018 Published: December 29, 2018

Copyright (c) 2018 by authors and Scientific Research Publishing Inc. This work is licensed under the Creative Commons Attribution International License (CC BY 4.0).

http://creativecommons.org/licenses/by/4.0/

c) (i) Open Access

\begin{abstract}
The variational statement of synthesis problem is generalized in order to account the additional requirements to the synthesized radiation pattern (RP) and field distribution in the specified points of near zone. For this aim, the minimizing functional is supplemented by term providing the possibility to minimize the values of field in these points; creating the deep zeros in the RP for the certain angular coordinates is realized too. The approach foresees reduction of an explicit formula for field values in a near zone. The results of computational modeling testify the possibility to create zeros in the given RP and to minimize the values of field in a near zone of plane arrays in a great extent.
\end{abstract}

\section{Keywords}

Synthesis Problem, Variational Statement, Plane Array, Near Zone, Euler's Equation, Numerical Modeling

\section{Introduction}

A lot of modern antenna systems are functioning by condition of a different series of various requirements to their radiation characteristics. One of such requirements is forming the deep minimums or zeros in the given points of near or far zones of antenna. This requirement corresponds to issues of the electromagnetic compatibility (EMC). Of course, all antenna systems are working at the condition that is characterized by the existence of various types of radio electronic systems and devices in its neighboring environment. Many engineering problems appear in this connection. The solution to these problems should provide the effective functioning neighbor antenna radiating systems that are placed 
closely. Therefore, the need for solving two interrelated problems of EMC appears. They are distribution of frequencies while working in the assigned frequency range, and a constructive improvement of the radio electronic system's individual elements.

The EMC problems were stated and discussed since $80^{\text {th }}$ of last century. In this regard, it should be noted pioneer monograph [1], and many journal papers (see, for example [2] [3] [4] [5] and references therein). The last papers appurtenant to this problem show that the development of the methods and means for providing the certain requirements to the field characteristics of antenna systems in the near and far zones is actual now. This leads to necessity of development of the modeling tools and respective software for solving the practical engineering problems related to EMC of antenna systems.

Some general recommendations how to improve EMC issues for radiating systems are set out in [6]. Different technical solutions and measurements for improving the EMC requirements in the neighboring environment are discussed in papers [7] [8] [9]. Application of numerical techniques in order to provide the series of restrictions on the radiation characteristics of antennas on the whole [10] [11], to RP only [12] [13] or to characteristics radiation in a near zone [14] gives possible to elaborate the general vision to EMC problem solving. However, a comprehensive solution to the EMC problem for radiating systems and antennas is far from complete. The generalized variational approach for solving the array's synthesis problems, proposed in this paper, is one of the means that allows to solve this problem at a more fundamental level. The mathematical statement of synthesis problem including the EMC specifications for plane arrays and results of modeling are discussed in the presented paper.

The paper is organized as follows.

The generalization of usual synthesis problem for plane array allowing to prescribe the restrictions on the radiation characteristics in a near zone is proposed and explanations to terms of formulated functional are given in Section 2. The formula for RP of array (array factor) is discussed with introducing the generalized angular coordinates. In Section 3, the procedure of obtaining the nonlinear integral equation for optimal distribution of currents in array elements, corresponding to minimizing functional, is described. Reduction of explicit formula for components of electromagnetic (EM) field in a near zone of array is made in Section 4. Section 5 and Section 6 are devoted to methods of creating zeros (deep gaps) in RP of array and values of EM field in a near zone. The computational results of modeling for rectangular and hexagonal arrays are presented and discussed in Sections 7; Section 8 contains concluding remarks and proposal for the future investigations.

\section{Variational Statement of Synthesis Problem}

The functional aimed to take into account a series of requirements to both the $\mathrm{RP}$ and field distribution in a near zone is formulated as sum of several terms [15] 
[16] [17], each of them ensures the best approximation to the array's radiation characteristics [18]

$$
\begin{aligned}
\sigma= & \int_{0}^{\pi} \int_{0}^{2 \pi} p(\theta, \varphi)[F(\theta, \varphi)-|f(\theta, \varphi)|]^{2} \mathrm{~d} \varphi \mathrm{d} \theta \\
& +\int_{0}^{\pi} \int_{0}^{2 \pi} q\left(\theta^{\prime}, \varphi^{\prime}\right)\left[U\left(\theta^{\prime}, \varphi^{\prime}\right)-c\left|u\left(\theta^{\prime}, \varphi^{\prime}\right)\right|\right]^{2} \mathrm{~d} \varphi^{\prime} \mathrm{d} \theta^{\prime} \\
& +\sum_{n=-N, m=-M}^{N, M} t_{n m}\left|I_{n m}\right|^{2}
\end{aligned}
$$

function $F(\theta, \varphi)$ is prescribed amplitude RP (by the condition of problem, it is real and positive), $|f(\theta, \varphi)|$ characterizes the module of RP created (synthesized) by array, $p(\theta, \varphi) \geq 0$ is a weight function that aimed to formulate specific demands to the module of $f(\theta, \varphi)$, in particular, to create zeros.

The second term in (1) provides an opportunity to minimize the values of field's amplitude in the fixed points of array's near zone. Such an assignment is fulfilled by means of increasing the $q\left(\theta^{\prime}, \varphi^{\prime}\right)$ value in the above points in comparison with the rest of them. Value $U\left(\theta^{\prime}, \varphi^{\prime}\right)$ is amplitude of given field in a near zone, by this $u\left(\theta^{\prime}, \varphi^{\prime}\right)$ is field created by array. The value of additional optimizing parameter $c$ is determined as $\mathrm{d} \sigma / \mathrm{d} c=0$.

The last term in (1) is applied to restrict the amplitude of currents in the array's elements. This provides to solve the problem to make away with the effect of super-directivity [19] [20] [21] for array. The additional optimizing values $t_{n m}$ are applied to restrict the amplitude of currents in the array's radiators.

We use for RP (array factor) of rectangular array the next formula [18]

$$
f\left(s_{1}, s_{2}\right)=A \mathbf{I} \equiv \sum_{m=-M_{1}}^{M_{1}} \sum_{n=-N_{1}}^{N_{1}} f_{n m}\left(s_{1}, s_{2}\right) I_{n m} \mathrm{e}^{i\left(c_{1} n s_{1}+c_{2} m s_{2}\right)},
$$

the RP of hexagonal array is determined similarly [22]. $I_{n m}$ are the currents in array's radiators, $f_{n m}\left(s_{1}, s_{2}\right)$ are the RPs of separate radiators.

We will use below the generalized angular coordinates $s_{1}, s_{2}$ instead of usual $\theta, \varphi$. This provides ability to present the dependence of RP of array on the one generalized parameter that includes the geometrical size of array and length of wave or frequency simultaneously. Such coordinates are $s_{1}=\sin \theta \cos \varphi / \sin \alpha_{1}$, $s_{2}=\sin \theta \sin \varphi / \sin \alpha_{2} \quad$ where $c_{1}=k d_{1} \sin \alpha_{1}, \quad c_{2}=k d_{2} \sin \alpha_{2}, k=2 \pi / \lambda$ is wavenumber, $\lambda$ is length of wave, values $d_{1}$ and $d_{2}$ correspond to distance between elements of array in $O x$ and $O y$ directions. It is assumed that the given RP $F$ differs from zero within the angles $\left[ \pm \alpha_{1}, \pm \alpha_{2}\right]$.

\section{Numerical Minimization of Functional}

In order to find the minimum of (1), we apply the approach of the variation calculus that yields in receiving the formula for the gradient of functional. Using such formula, we pass to non-linear integral equation [18] with respect to unknown currents in the array's elements.

The notations can be simplified in a great extent if to use the operator form as 
$f\left(s_{1}, s_{2}\right)=A \mathbf{I}$ for synthesized RP. For this goal, the notion of adjoint operators $A^{*}$ and $B^{*}$ is applied too.

The $n m$-th component for gradient of (1) on the currents $I_{n m}$ can be presented as

$$
\begin{aligned}
z_{n m}= & t I_{n m}+A^{*}\left[p\left(A I_{n m}-F \mathrm{e}^{i \arg A I_{n m}}\right)\right] \\
& +B^{*}\left[q\left(c^{2} B I_{n m}-c U \mathrm{e}^{i \arg B I_{n m}}\right)\right],\left(m=\overline{-M_{1}, M_{1}}, n=\overline{-N_{1}, N_{1}}\right) .
\end{aligned}
$$

Here, operator $B$ presents explicit relation for the field's components in a near zone. It is determined similarly to $A$, the difference is that the additional multiplier, depending on the distance $r$ to array is presented. Equating gradient to zero, we receive the non-linear vector-matrix equation for the optimal currents in array's elements

$$
t \mathbf{I}+A^{*}\left[p\left(A \mathbf{I}-F \mathrm{e}^{i \arg A \mathbf{I}}\right)\right]+B^{*}\left[q\left(c^{2} B \mathbf{I}-c U \mathrm{e}^{i \arg B \mathbf{I}}\right)\right]=0 .
$$

In practice, we do not deal with solving (4) because in the iterative procedure of its solving there is necessary to calculate the actions of $A, B, A^{*}$ and $B^{*}$ operators. If one to act on (4) by operator $A$, we have the nonlinear integral equation of Hammerstein's type with respect to RP $f[18]$, because the $A A^{*}(\cdot)$, $A B^{*}(\cdot)$ are included into respective exponent.

The explicit relation for operator $A^{*}$ is [18]

$$
\left[A^{*} f\right]_{n m}=\frac{c_{1} c_{2}}{4 \pi^{2}} \int_{-1}^{1} \int_{-1}^{1} f\left(s_{1}, s_{2}\right) f_{n m}^{*}\left(s_{1}, s_{2}\right) \times \mathrm{e}^{-i\left(c_{1} n s_{1}+c_{2} m s_{2}\right)} \mathrm{d} s_{1} \mathrm{~d} s_{2} .
$$

In the last formula procedure of integration in limits $[0,2 \pi]$ and $[0, \pi]$ is replaced by twice integration over $[-1,1]$. This is result of use of new coordinates $s_{1}$ and $s_{2}$ instead of initial $\theta$ and $\varphi$. Determination of operator $B^{*}$ is realized similarly.

If one to use the subsequent application of $A, A^{*}$, as well as $B, B^{*}$ operators and operator form (2) of RP, the numerical calculations are much simple. This allows to receive the nonlinear integral equation relatively RP $f\left(s_{1}, s_{2}\right)$ and explicit formulas for the kernel of this equation [18].

\section{Determination of Field Components in Near Zone}

In order to carry out with explicit relation for the field components in a near zone using the currents $I_{n m}$ in the radiators of array, formula for the electric vector potential [23]

$$
\vec{A}_{F r}^{e}(r, \theta, \varphi)=\frac{\mathrm{e}^{-i k r}}{4 \pi R} \int_{V} I\left(x^{\prime}, y^{\prime}, z^{\prime}\right) \mathrm{e}^{i k\left[r^{\prime} \cos \alpha+\frac{r^{\prime 2}}{2 r}\left(1-\cos ^{2} \alpha\right)\right]} \mathrm{d} V
$$

is applied. Here

$$
R \approx r\left[1-\frac{r^{\prime}}{r} \cos \alpha+\frac{r^{\prime 2}}{2 r^{2}}\left(1-\cos ^{2} \alpha\right)\right]
$$

that is we will restrict ourselves to the value of order $O\left(1 / r^{2}\right)$. In the case of 
plane array

$$
r^{\prime} \cos \alpha=x^{\prime} \sin \theta \cos \varphi+y^{\prime} \sin \theta \sin \varphi
$$

so far as the array is placed in the $x^{\prime} O y^{\prime}$ plane.

For plane array, $\vec{A}_{F r}^{e}(r, \theta, \varphi)$ can be written as

$$
\begin{aligned}
\vec{A}_{F r}^{e}(r, \theta, \varphi)= & \frac{\mathrm{e}^{-i k r}}{4 \pi R} \sum_{m=-M_{1}}^{M_{1}} \sum_{n=-N_{1}}^{N_{1}} f_{n m}\left(s_{1}, s_{2}\right) \vec{I}_{n m} \\
& \times \exp \left[i\left(n k d_{1} \sin \theta \cos \varphi+m k d_{2} \sin \theta \sin \varphi\right)\right] v_{n m}\left(r, r^{\prime}, \theta, \varphi\right),
\end{aligned}
$$

where

$$
v_{n m}\left(r, r^{\prime}, \theta, \varphi\right)=\exp \left[i\left(x_{n m}^{2}+y_{n m}^{2}-\left(x_{n m} \sin \theta \cos \varphi+y_{n m} \sin \theta \sin \varphi\right)^{2}\right) / 2 r\right] .
$$

Here $\vec{I}_{n m}$ represent the vector current distributions of array's elements; by this radius $r^{\prime}$ is replaced by $\sqrt{x_{n m}^{2}+y_{n m}^{2}}$ for all array's radiators. Without loss of generality, one can assume the separation for $\vec{I}_{n m}$ currents onto two axes $O x^{\prime}$ and $O y^{\prime}$, and to consider values of $\vec{A}_{F r}^{e}(r, \theta, \varphi)$ for respective components independently.

Following the above assumptions, we will accept the same relations for the $E$ and $H$ field components in a near zone. As result, we have

$$
\begin{array}{rrr}
H_{r}=0 & H_{\theta}=i k A_{\varphi}^{e} & H_{\varphi}=-i k A_{\theta}^{e} \\
E_{r}=0 & E_{\theta}=-i \omega \mu A_{\theta}^{e} & E_{\varphi}=-i \omega \mu A_{\varphi}^{e}
\end{array}
$$

In such a way, we have obtained relations (9), (11) or (9), (12) for determination $E$ and $H$ field components. These subsequent formulas can be considered as the linear operator $B$. This is because of linear dependence of $E$ and $H$ on the distribution of currents $\vec{I}_{n m}$. In such consideration, operator $B^{*}$ is determined by introducing the respective Hilbertian space, as it was done to obtain $A^{*}$ [24].

\section{Creation of Zeros in the RP}

We rewrite (2) as

$$
f\left(s_{1}, s_{2}\right)=A \mathbf{I} \equiv \sum_{m=-M_{1}}^{M_{1}} \sum_{n=-N_{1}}^{N_{1}} f_{n m}\left(s_{1}, s_{2}\right) I_{n m} \mathrm{e}^{i\left(c_{1} d_{n} s_{1}+c_{2} d_{m} s_{2}\right)},
$$

where

$$
\begin{aligned}
& d_{n}=n-\left(N_{1}+1-\delta_{1} / 2\right), N_{1}=\operatorname{entier}(N / 2), \\
& d_{m}=m-\left(M_{1}+1-\delta_{2} / 2\right), M_{1}=\operatorname{entier}(M / 2) .
\end{aligned}
$$

Function $f\left(s_{1}, s_{2}\right)$ is periodical, namely

$$
f\left(s_{1}+\frac{2 \pi \nu}{c_{1}}, s_{2}+\frac{2 \pi \mu}{c_{2}}\right)=\mathrm{e}^{\mathrm{i} \pi\left(\delta_{1} v+\delta_{2} \mu\right)} f\left(s_{1}, s_{2}\right),
$$

that is important for consideration below.

Formulas (13)-(15) are used for presentation of RP using the Kotelnikov's formula [25] and forming zeros in the given points $\left(s_{1}^{*}, s_{2}^{*}\right)$. For this goal, we 
present (13) as

$$
f\left(s_{1}, s_{2}\right)=\sum_{n=1}^{N} \varphi_{n}\left(s_{2}\right) \mathrm{e}^{i c_{1} d_{n} s_{1}}
$$

where

$$
\varphi_{n}\left(s_{2}\right)=\sum_{m=1}^{M} I_{n m} \mathrm{e}^{i c_{2} d_{m} s_{2}}
$$

We use the greed

$$
\begin{aligned}
& \left\{\Delta_{1}=\frac{2 \pi}{c_{1} L_{1}}, \Delta_{2}=\frac{2 \pi}{c_{2} L_{2}}\right\}, L_{1} \geq N, L_{2} \geq M \text { in the region } \\
& \Omega=\left\{\left[-\pi / c_{1}, \pi / c_{1}\right] \times\left[-\pi / c_{2}, \pi / c_{2}\right] \text { of } s_{1} \text { and } s_{2}\right. \text { variables. }
\end{aligned}
$$

Kotelnikov's formula for (17) is

$$
\bar{f}_{n}\left(s_{2}\right)=\frac{1}{L_{2}} \sum_{d_{\mu}=1}^{L_{2}} \varphi_{n}\left(s_{2}^{(\mu)}\right) \frac{\sin \left[L_{2} c_{2}\left(s_{2}-s_{2}^{(\mu)}\right) / 2\right]}{\sin \left[c_{2}\left(s_{2}-s_{2}^{(\mu)}\right) / 2\right]},
$$

where $s_{2}^{(\mu)}=d_{\mu} \Delta_{2}$.

Substituting (18) into (13), we receive

$$
f\left(s_{1}, s_{2}\right)=\frac{1}{L_{2}} \sum_{d_{\mu}=1}^{L_{2}} \frac{\sin \left[L_{2} c_{2}\left(s_{2}-s_{2}^{(\mu)}\right) / 2\right]}{\sin \left[c_{2}\left(s_{2}-s_{2}^{(\mu)}\right) / 2\right]} \times \sum_{n=1}^{N} \varphi_{n}\left(s_{2}^{(\mu)}\right) \mathrm{e}^{i c_{1} d_{n} s_{1}} .
$$

Finally, the Kotelnikov's formula for plane array is

$$
\begin{aligned}
\bar{f}\left(s_{1}, s_{2}\right)= & \frac{1}{L_{1} L_{2}} \sum_{d_{v}=1}^{L_{1}} \sum_{d_{\mu}=1}^{L_{2}} f_{0}\left(s_{1}^{(v)}, s_{2}^{(\mu)}\right) \times \frac{\sin \left[L_{1} c_{1}\left(s_{1}-s_{1}^{(v)}\right) / 2\right]}{\sin \left[c_{1}\left(s_{1}-s_{1}^{(v)}\right) / 2\right]} \\
& \cdot \frac{\sin \left[L_{2} c_{2}\left(s_{2}-s_{2}^{(\mu)}\right) / 2\right]}{\sin \left[c_{2}\left(s_{2}-s_{2}^{(\mu)}\right) / 2\right]},
\end{aligned}
$$

$s_{1}^{(v)}=d_{v} \Delta_{1}, f_{0}\left(s_{1}^{(v)}, s_{2}^{(\mu)}\right)$ are the values of initial PR in the interpolation points. Of course,

$$
\bar{f}\left(s_{1}^{(v)}, s_{2}^{(\mu)}\right)=f_{0}\left(s_{1}^{(v)}, s_{2}^{(\mu)}\right) .
$$

Let us minimization point $\left(s_{1}^{*}, s_{2}^{*}\right)$ coincides with one of interpolation point, as example $\left(s_{1}^{\left(\nu_{0}\right)}, s_{2}^{\left(\mu_{0}\right)}\right)$. We determine the function $\bar{f}\left(s_{1}^{(v)}, s_{2}^{(\mu)}\right)$ in the interpolation points as

$$
\bar{f}\left(s_{1}^{(v)}, s_{2}^{(\mu)}\right)= \begin{cases}0, & \text { if } v=v_{0}, \mu=\mu_{0}, \\ f_{0}\left(s_{1}^{(v)}, s_{2}^{(\mu)}\right), & \text { if } v \neq v_{0}, \mu \neq \mu_{0} .\end{cases}
$$

The problem to synthesize zero is formulated as

$$
\sigma=\left\|\bar{f}-f^{*}\right\|=\iint_{\Omega}\left|\bar{f}\left(s_{1}, s_{2}\right)-f^{*}\left(s_{1}, s_{2}\right)\right|^{2} \mathrm{~d} s_{1} \mathrm{~d} s_{2},
$$

where $f^{*}\left(s_{1}, s_{2}\right)$ is RP with formed zero.

The condition of stationarity of (23) leads to formula for currents $I_{n m}^{*}$ 


$$
I_{n m}^{*}=\frac{c_{1} c_{2}}{4 \pi^{2}} \iint_{\Omega} f(s, s) \mathrm{e}^{-i\left(c_{1} d_{n} s_{1}+c_{2} d_{m} s_{2}\right)} \mathrm{d} s_{1} \mathrm{~d} s_{2}, \quad(n=\overline{1, N}, m=\overline{1, M}) .
$$

In fact, values (24) are the coefficients of discrete Fourier transform of function $f\left(s_{1}, s_{2}\right)$. More simple formula for optimal currents $I_{n m}^{*}$ is [18]

$$
I_{n m}^{*}=I_{n m}^{0}-\frac{f\left(s_{1}^{\left(\nu_{0}\right)}, s_{2}^{\left(\mu_{0}\right)}\right)}{L_{1} L_{2}} \mathrm{e}^{-i\left[c_{1} d_{\nu} s_{1}^{\left(\nu_{0}\right)}+c_{2} d_{\mu} s_{2}^{\left(\mu_{0}\right)}\right]} .
$$

In the case, when point $\left(s_{1}^{*}, s_{2}^{*}\right)$ does not coincide with any interpolation point and $L_{1}>N, L_{2}>M$, optimal $I_{n m}^{*}$ can be determined by the iterative process

$$
I_{n m}^{*(j+1)}=I_{n m}^{*(J)}-\frac{f^{(j)}\left(s_{1}^{\left(\nu_{0}\right)}, s_{2}^{\left(\mu_{0}\right)}\right)}{L_{1} L_{2}} \mathrm{e}^{-i\left[c_{1} d_{\nu} s_{1}^{\left(\nu_{0}\right)}+c_{2} d_{\mu} s_{2}^{\left(\mu_{0}\right)}\right]},
$$

where $j$ is iteration number.

Iterative process (26) converges because [26]

$$
\left|f^{(j+1)}\left(s_{1}^{\left(\nu_{0}\right)}, s_{2}^{\left(\mu_{0}\right)}\right)\right|<\left|f^{(j)}\left(s_{1}^{\left(\nu_{0}\right)}, s_{2}^{\left(\mu_{0}\right)}\right)\right|\left(1-\frac{N M}{L_{1} L_{2}}\right) .
$$

\section{Creation of Zeros in a Near Zone}

Let us $E_{\varphi}\left(\theta^{\prime}, \varphi^{\prime}\right)=u\left(\theta^{\prime}, \varphi^{\prime}\right)$. The weight function $q\left(\theta^{\prime}, \varphi^{\prime}\right)$ is used to create zero in the certain point $\left(\theta^{*}, \varphi^{*}\right)$ of near zone. As a rule, neighbor $\left(\theta^{*} \pm \Delta \theta, \varphi^{*} \pm \Delta \varphi\right)$ of this point is used, and integration within $[0, \pi] \times[0,2 \pi]$ is replaced by integration within $\left[\theta^{*} \pm \Delta \theta, \varphi^{*} \pm \Delta \varphi\right]$.

Minimizing (1), we minimize $E_{\varphi}\left(\theta^{\prime}, \varphi^{\prime}\right)$ too, and we have optimal currents $I_{n m}^{*}$ [27]. The components of electric vector potential (9) are determined by known $I_{n m}^{*}$. After this, we determine the rest of components of EM field. The radiation power density (RPD) is determined as [22]

$$
W_{a v}(x, y, z)=\frac{1}{2} \operatorname{Re}\left[\mathbf{E} \times \mathbf{H}^{*}\right]\left(\mathrm{W} / \mathrm{m}^{2}\right) .
$$

\section{Computational Modeling}

\subsection{Creating Zeros in RP}

Firstly, we show the computational results for the hexagonal array that has 127 elements. By this we assume known RPs $f_{n m}\left(s_{1}, s_{2}\right)$ of array radiators. The computations are carried out using free MathWorks software.

Results are shown for frequency equal to $11.99 \mathrm{GHz}$, distance between radiators in the linear subarrays is equal to $0.0078 \mathrm{~m}$, and distance between the linear subarrays is equal to $0.0087 \mathrm{~m}$. The prescribed amplitude RP $F \equiv 1$ in the given range $F \equiv 1$ (Figure 1). In Figure 2, Figure 3, the results that show forming zeros in the vicinity of the first sidelobe are shown for $E$-plane of array. Using the weight function $p(\theta, \varphi)$ in functional (1), we form zeros in points $\varphi=\pi / 2,3 \pi / 2$ and $\theta= \pm 26.4^{\circ}$. 


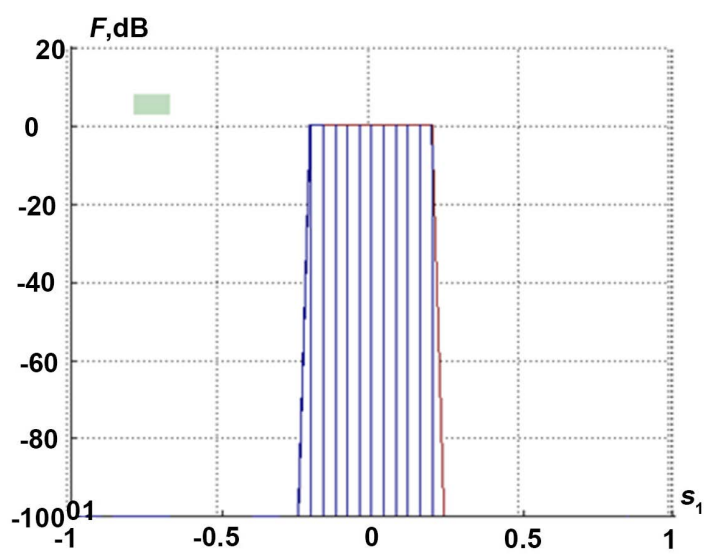

Figure 1. Front view of the prescribed RP.

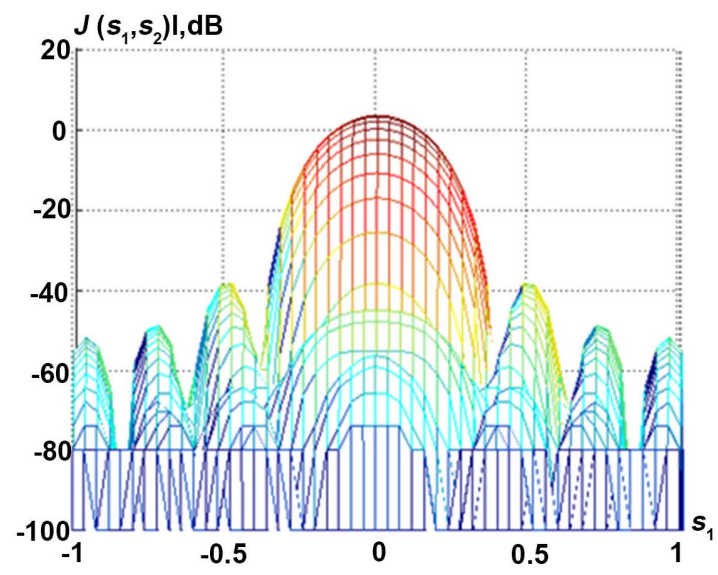

Figure 2. The initial synthesized amplitude RP.

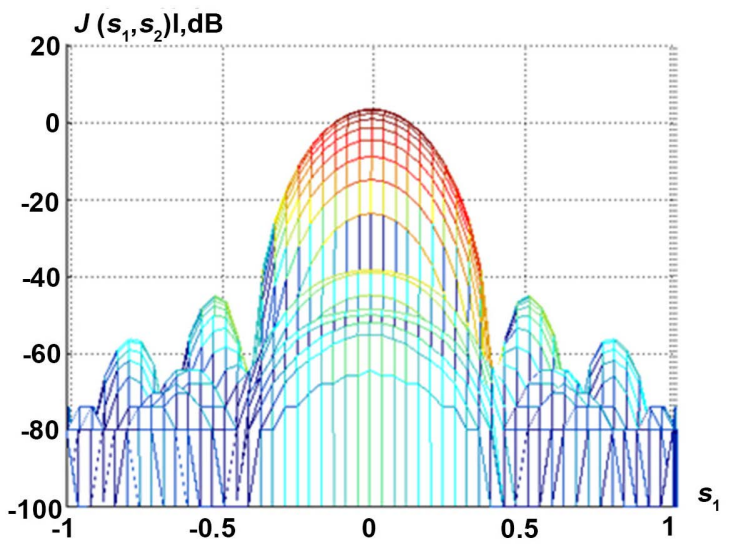

Figure 3. The synthesized RP with zero in the area of first sidelobes, $p=10.0$.

The level of the first sidelobe was reduced to $-52.6 \mathrm{~dB}$ (Figure 3). The obtained results testify that such a way to create zeros has some limitation. Use of Kotelnikov's procedure (25) or (26) is more complicate, but it can provide much small zeros. 


\subsection{Creating Zeros in a Near Zone}

Secondly, the results for antenna with rectangular placement of radiator are considered. Array that consists of 121 radiators is examined, and distance $d$ between them is equal to $0.01 \mathrm{~m}$, that is square of array is equal to $0.01 \mathrm{~m}^{2}$; the work frequency is equal to $5.976 \mathrm{GHz}$, by this the condition $d<\lambda / 2$ is fulfilled that allows to form the main lobe of RP without several maximums (see [22]). In Figure 4, values of the RPD are shown without forming zeros at the distance $0.5 \mathrm{~m}$ of array.

For this case, RPD changes from $0.16 \mathrm{~W} / \mathrm{m}^{2}$ to $0.207 \mathrm{~W} / \mathrm{m}^{2}$. The requirement to form zero was stated for the distance $r=0.5 \mathrm{~m}$ of array at the next points: $\theta= \pm \pi / 4, \varphi=\pi$. To obtain minimal values of RPD in these points, the field $U\left(\theta^{\prime}, \varphi^{\prime}\right)$ in (1) was equated to zero; herewith values of the weight function $q\left(\theta^{\prime}, \varphi^{\prime}\right)$ were decreased on one order greater in the comparison of rest values of $\theta^{\prime}$ and $\varphi^{\prime}$ angles.

Such modification of initial data allows to decrease the RPD from quantity of $0.1831 \mathrm{~W} / \mathrm{m}^{2}$ to quantity of $0.0373 \mathrm{~W} / \mathrm{m}^{2}$ that is $21.7 \%$ of its initial value. In the rest range of $\theta^{\prime}$ and $\varphi^{\prime}$ values, the RPD increased not more than on 10.2\% (see Figure 5). The obtained results testify that the field values and its structure outside the considered $\theta^{\prime}$ and $\varphi^{\prime}$ for such $r$ is close to constant value.

In Figure 6, the results related to minimization of the RPD in one point are shown. Similarly to the previous example, the RPR values diminish significantly.

If distance $r$ to array's plane increases the values of the RPD decrease quickly. The respective results are shown in Figure 7 for the distance that is equal to $r=10.0 \mathrm{~m}$ to array.

The presented results testify that the RPD diminishes in a great extent for such parameters of array; by this its values are more two order lower than for values corresponding to $r=0.5 \mathrm{~m}$. For example, the maximum of RPD for $r=6.0 \mathrm{~m}$ reaches $1.37 \times 10^{-2} \mathrm{~W} / \mathrm{m}^{2}$. It confirms that area of small values of the RPD is placed at distance equal about to $6.0 \mathrm{~m}$ from the plane of array. This is important data for the creating zeros for the procedure of design of such

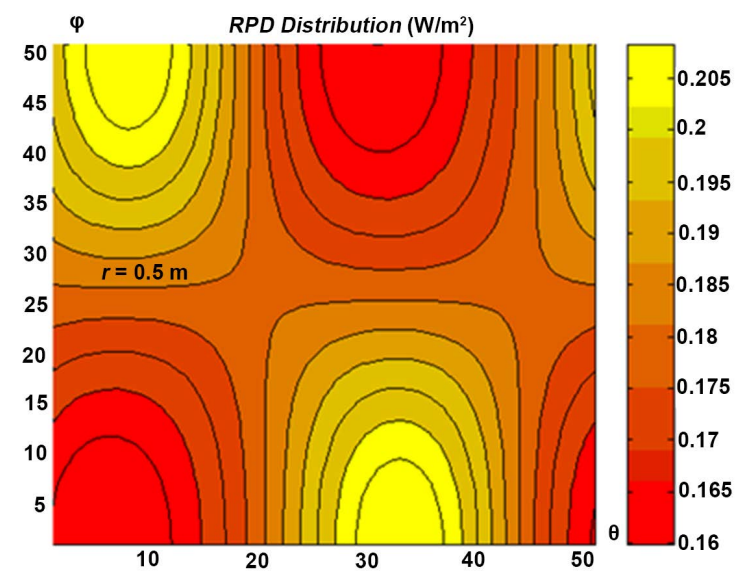

Figure 4. The initial distribution of the RPD at $r=0.5 \mathrm{~m}$. 


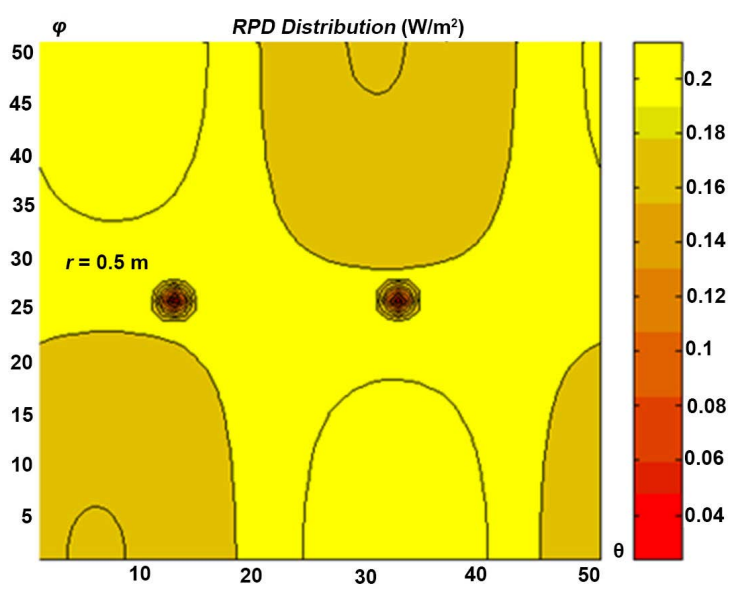

Figure 5. The RPD with forming zero in two points at $r=0.5 \mathrm{~m}$.

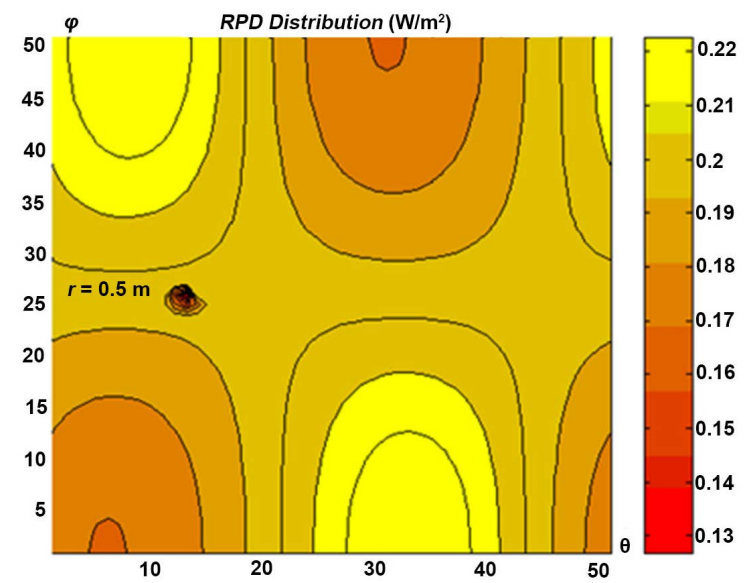

Figure 6. The RPD with forming zero in one point at $r=0.5 \mathrm{~m}$.

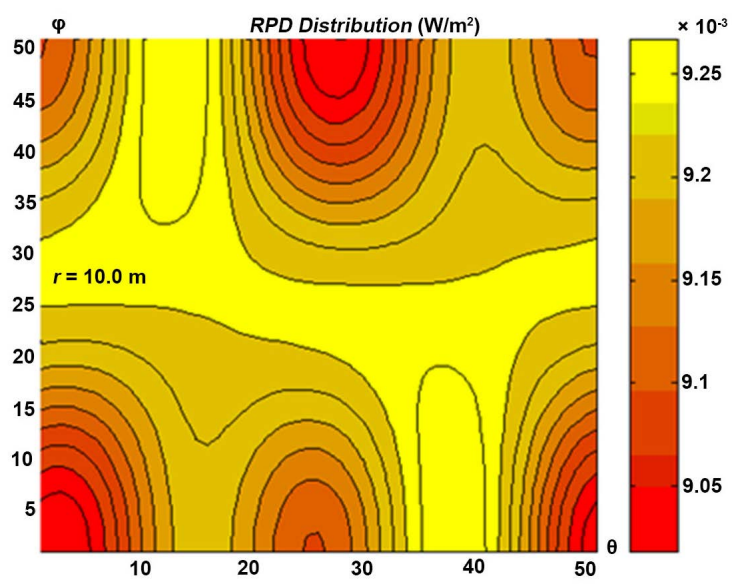

Figure 7. The RPD at the distance $10.0 \mathrm{~m}$.

type of arrays. The form and values of the main and sidelobes of the array's RP are varied few in comparison with the case when the synthesis problem is consi- 
dered without the creating of zeros. The main lobe is wider at level of 0.5 power of the RP on 4.7\%, and the level of sidelobes wherein increased on $3.7 \mathrm{~dB}$.

One more requirement to increase the array's performance consists of the ability to take into account the additional limitations on the amplitude and phase distribution of the currents $\vec{I}_{n m}$ in the array elements. This is can be carry out by the prescribing the weight multipliers $t_{n m}$ instead of fixed $t$ [28]. For goal to decrease $\vec{I}_{n m}$, the values $t_{n m}$ should be increased. The numerical calculations demonstrate that such a way is suitable to avoid the superdirectivity of array's elements to a large degree. The weight multipliers $t_{n m}$ are prescribed so that the amplitude of currents $\vec{I}_{n m}$ is close to constant distribution.

\section{Conclusions}

The variational approach for solving the antenna synthesis problem by the given amplitude RP is modified for the goal to take into account the additional restrictions on the array radiation in the near and far zones. The optimizing functional is formularized in such a way that the deep zeros can be created in the points of near and far zones.

The results of the numerical calculations demonstrate that the level of formed zeros less than $-80 \mathrm{~dB}$ in the area of far sidelobes of the RP can be reached. By this, the array's RP in the main lobe varies insignificantly, and difference of the obtained and initial RP's values does not exceed $3.7 \mathrm{~dB}$. The minimization of the field at the distance about $10.0 \mathrm{~m}$ from array does not lead to the considerable variation of the array's RP.

The future research in the topic under investigation is designated for consideration of vector character of array's RP and non-transversal character of EM field in a near zone of array, taking into account the mutual coupling of array's radiators, as well as at development of powerful numerical methods and respective software for modeling the array's radiation properties in the real physical environment.

\section{Conflicts of Interest}

The authors declare no conflicts of interest regarding the publication of this paper.

\section{References}

[1] Keiser, K.L. (2000) Electromagnetic Compatibility Handbook. CRC Press, Boca Raton, London, New York, Washington DC.

[2] Hansen, D. (2003) Review of EMC Main Aspects in Fast PLC Including Some History. 2003 IEEE International Symposium on Electromagnetic Compatibility (EMC '03), 1, 184-192. https://doi.org/10.1109/ICSMC2.2003.1428226

[3] Lezynski, P. (2018) Random Modulation in Inverters with Respect to Electromagnetic Compatibility and Power Quality. IEEE Journal of Emerging and Selected Topics in Power Electronics, 6, 782-790. https://doi.org/10.1109/JESTPE.2017.2787599 
[4] Lambrecht, N., Pues, H., De Zutter, D. and Ginste, D.V. (2017) Modeling of Contact Bounce in a Transient Electromagnetic Compatibility Test for the Analysis and Optimization of Nonlinear Devices. IEEE Transactions on Electromagnetic Compatibility, 59, 541-544. https://doi.org/10.1109/TEMC.2016.2618345

[5] Trainotti, V. (2017) Electromagnetic Compatibility (EMC) Antenna Gain and Factor. IEEE Transactions on Electromagnetic Compatibility, 59, 1006-1015. https://doi.org/10.1109/TEMC.2016.2642833

[6] Montrose, M.L. (2008) EMC and Propagating Fields. IEEE Antennas and Propagation Magazine, 50, 224-226. https://doi.org/10.1109/MAP.2008.4653720

[7] Liang, C.-H., Dang, X.-J., Wang, N. and Yuan, H.-B. (2011) Generalized Isolation between Antennas for EMC Problems in Complex EM Environments. IEEE Transactions on Electromagnetic Compatibility, 53, 645-652.

https://doi.org/10.1109/TEMC.2011.2140375

[8] Foged, L.J., Scialacqua, L., Mioc, F., Saccardi, F., Iversen, P.O., Shmidov, L., Braun, R., Quijano, J.L.A. and Vecchi, G. (2013) Echo Suppression by Spatial-Filtering Techniques in Advanced Planar and Spherical Near-Field Antenna Measurements [AMTA Corner]. IEEE Antennas and Propagation Magazine, 55, 235-242. https://doi.org/10.1109/MAP.2013.6735525

[9] Perez, J.R. and Basterrechea, J. (2007) Comparison of Different Heuristic Optimization Methods for Near-Field Antenna Measurements. IEEE Transactions on Antennas and Propagation, 55, 549-555. https://doi.org/10.1109/TAP.2007.891508

[10] Bogdanov, F.G., Karkashadze, D.D., Jobava, R.G., Gheonjian, A.L., Yavolovskaya, E.A., Bondarenko, N.G. and Ullrich, C. (2010) Validation of Hybrid MoM Scheme with Included Equivalent Glass Antenna Model for Handling Automotive EMC Problems. IEEE Transactions on Electromagnetic Compatibility, 52, 164-172. https://doi.org/10.1109/TEMC.2009.2036003

[11] Parrikar, R.P. and Gupta, K.C. (1998) Multiport Network Model for CAD of Electromagnetically Coupled Microstrip Patch Antennas. IEEE Transactions on Antennas and Propagation, 46, 475-483. https://doi.org/10.1109/8.664110

[12] Foged, L.J., Scialacqua, L., Saccardi, F., Quijano, J.L.A. and Vecchi, G. (2014) Application of the Dual-Equation Equivalent-Current Reconstruction to Electrically Large Structures by Fast Multipole Method Enhancement [AMTA Corner]. IEEE Antennas and Propagation Magazine, 56, 264-273.

https://doi.org/10.1109/MAP.2014.6971966

[13] Ferrieres, X., Parmantier, J.-P., Bertuol, S. and Ruddle, A.R. (2004) Application of a Hybrid Finite Difference/Finite Volume Method to Solve an Automotive EMC Problem. IEEE Transactions on Electromagnetic Compatibility, 46, 624-634. https://doi.org/10.1109/TEMC.2004.837837

[14] Quijano, J.L.A. and Vecchi, G. (2010) Near- and Very Near-Field Accuracy in 3-D Source Reconstruction. IEEE Antennas and Wireless Propagation Letters, 9, 634-637. https://doi.org/10.1109/LAWP.2010.2055032

[15] Voitovich, N.N. (1985) Synthesis of Plane Closed Antenna with the Field Restrictions in the Near Zone. Radiotechnika i Elektronika, 30, 458-462. (In Russian)

[16] Schnattinger, G., Mauermayer, R.A.M. and Eibert, T.F. (2014) Monostatic Radar Cross Section Near-Field Far-Field Transformations by Multilevel Plane-Wave Decomposition. IEEE Transactions on Antennas and Propagation, 62, 4259-4268. https://doi.org/10.1109/TAP.2014.2323429

[17] Galindo-Israel, V. and Rahmat-Samii, Y. (1981) A New Look at Fresnel Field Computation Using the Jacobi-Bessel Series. IEEE Transactions on Antennas and 
Propagation, 29, 885-898. https://doi.org/10.1109/TAP.1981.1142680

[18] Andriychuk, M.I., Voitovich, N.N., Savenko, P.A. and Tkachuk, V.P. (1993) Antenna Synthesis According to the Amplitude Radiation Pattern. Naukova Dumka, Kiev. (In Russian)

[19] Salt, H. (1977) Practical Realization of Superdirective Arrays. Radio and Electronic Engineer, 47, 143-156. https://doi.org/10.1049/ree.1977.0021

[20] Yang, T.C. (2018) Performance Analysis of Superdirectivity of Circular Arrays and Implications for Sonar Systems. IEEE Journal of Oceanic Engineering, 43, 1-11. https://doi.org/10.1109/JOE.2018.2801144

[21] Diao, J. and Warnick, K.F. (2018) Practical Superdirectivity with Resonant Screened Apertures Motivated by a Poynting Streamlines Analysis. IEEE Transactions on Antennas and Propagation, 66, 432-437. https://doi.org/10.1109/TAP.2017.2772929

[22] Balanis, C.A. (1997) Antenna Theory: Analysis and Design. John Wiley \& Sons, New York, Chichester, Brisbane, Toronto, Singapore.

[23] Markov, G.T., Petrov, B.M. and Grudinskaya, G.P. (1979) Electrodinamics and Propagation of Radiowaves. Sov. Radio, Moscow. (In Russian)

[24] Andriychuk, M.I. (2000) Investigation of Solution of the Nonlinear Synthesis Problem for the Waveguide Array. International Seminar/ Workshop on Direct and Inverse Problems of Electromagnetic and Acoustic Wave Theory, Tbilisi, 47-51.

[25] Kotelnikov, V.A. (2006) On the Capacity of "Ether" and Wire in Electro-Communication (Appendix). UFN, 176, 762-770. https://doi.org/10.3367/UFNr.0176.200607h.0762

[26] Andriychuk, M.I. and Savenko, P.O. (2000) Synthesis of a Waveguide Array with Due Regard for the Mutual Coupling of Radiarors. International Conference on Mathematical Methods in Electromagnetic Theory, Kharkiv, 12-15 September 2000, Vol. 2, 604-606.

[27] Savenko, P.O. and Anokhin, V.J. (1997) Synthesis of Amplitude-Phase Distribution and Shape of Plane Antenna Aperture for a Given Power Pattern. IEEE Transactions on Antennas and Propagation, 45, 744-747. https://doi.org/10.1109/8.564102

[28] Andriychuk, M.I. and Voitovich, N.N. (2013) Antenna Synthesis According to Power Radiation Parttern with Condition of Norm Equality. International Seminarl Workshop on Direct and Inverse Problems of Electromagnetic and Acoustic Wave Theory, Lviv, September 23-26 2013, 137-140. 